\title{
WEAK TYPE ESTIMATES FOR SINGULAR INTEGRALS RELATED TO A DUAL PROBLEM OF MUCKENHOUPT-WHEEDEN
}

\author{
ANDREI K. LERNER, SHELDY OMBROSI, AND CARLOS PÉREZ
}

\begin{abstract}
A well known open problem of Muckenhoupt-Wheeden says that any Calderón-Zygmund singular integral operator $T$ is of weak type $(1,1)$ with respect to a couple of weights $(w, M w)$. In this paper we consider a somewhat "dual" problem:

$$
\sup _{\lambda>0} \lambda w\left\{x \in \mathbb{R}^{n}: \frac{|T f(x)|}{M w}>\lambda\right\} \leq c \int_{\mathbb{R}^{n}}|f| d x .
$$

We prove a weaker version of this inequality with $M^{3} w$ instead of $M w$. Also we study a related question about the behavior of the constant in terms of the $A_{1}$ characteristic of $w$.
\end{abstract}

\section{INTRODUCTION}

In 1971, C. Fefferman and E.M. Stein [8] established the following extension of the classical weak-type $(1,1)$ property of the HardyLittlewood maximal operator $M$ :

$$
\sup _{\lambda>0} \lambda w\left\{x \in \mathbb{R}^{n}: M f(x)>\lambda\right\} \leq c \int_{\mathbb{R}^{n}}|f| M w d x,
$$

where a weight $w$ is supposed to be a non-negative locally integrable function and $w(E)=\int_{E} w(x) d x$.

Assume now that $T$ is a Calderón-Zygmund singular integral operator. It was conjectured by B. Muckenhoupt and R. Wheeden [13] many years ago that the full analogue of (1.1) holds for $T$, namely,

$$
\sup _{\lambda>0} \lambda w\left\{x \in \mathbb{R}^{n}:|T f(x)|>\lambda\right\} \leq c \int_{\mathbb{R}^{n}}|f| M w d x .
$$

This problem is open even for the Hilbert transform. In this direction, the following result can be found in [16]:

2000 Mathematics Subject Classification. 42B20, 42B25.

Key words and phrases. Calderón-Zygmund operators, Weights.

The first is supported by the Spanish Ministery of Education under the program "Programa Ramón y Cajal, 2006. The second author is supported by a fellowship from the same institution. All the authors also supported by the same institution with research grant MTM2006-05622. 
Theorem 1.1. There is a constant $c=c(n, T)$ such that for any weight $w$ and for all $f$,

$$
\sup _{\lambda>0} \lambda w\left\{x \in \mathbb{R}^{n}:|T f(x)|>\lambda\right\} \leq c \int_{\mathbb{R}^{n}}|f| M^{2} w d x .
$$

Here $M^{k}$ denotes the operator $M$ iterated $k$ times. In fact, it is shown in [16] that $M^{2}$ can be replaced by the (pointwise) smaller operator $M^{1+\varepsilon} w$ for any $\varepsilon>0$ (see Remark 4.1 below for the definition of $M^{\alpha} w$, $\alpha \geq 1$ ).

We claim that (1.2) has a somehow "dual" version, namely,

$$
\sup _{\lambda>0} \lambda w\left\{x \in \mathbb{R}^{n}: \frac{|T f(x)|}{M w}>\lambda\right\} \leq c \int_{\mathbb{R}^{n}}|f(x)| d x .
$$

Indeed, if conjecture (1.2) holds, say for the Hilbert transform $H$, then by the extrapolation theorem from [6] we can derive the following inequality for any $1<p<\infty$ :

$$
\int_{\mathbb{R}}|H f(x)|^{p} w d x \leq c \int_{\mathbb{R}}|f(x)|^{p}\left(\frac{M w}{w}\right)^{p} w d x .
$$

Then by duality we have that for any $1<p<\infty$,

$$
\int_{\mathbb{R}}\left(\frac{|H f(x)|}{M w}\right)^{p^{\prime}} w d x \leq c \int_{\mathbb{R}}\left(\frac{|f(x)|}{w}\right)^{p^{\prime}} w d x,
$$

and hence (1.3) can be viewed as a limiting weak-type $(1,1)$ case of the latter inequality.

Estimates of the sort (1.3) are called sometimes in the literature mixed weak type. They appeared for the first time in the work of B. Muckenhoupt and R. Wheeden [14] and later on in Sawyer's work [17]. More recently, extensions of these results can be found in [5] and [15].

We do not know how to prove (1.3) even for $M^{2} w$ instead of $M w$. However we prove the following result.

Theorem 1.2. There is a constant $c=c(n, T)$ such that for any weight $w$ and for all $f \in L^{1}\left(\mathbb{R}^{n}\right)$,

$$
\sup _{\lambda>0} \lambda w\left\{x \in \mathbb{R}^{n}: \frac{|T f(x)|}{M^{3} w}>\lambda\right\} \leq c \int_{\mathbb{R}^{n}}|f| d x .
$$

It is interesting to observe that even a weak variant of (1.2) is not known:

$$
\sup _{\lambda>0} \lambda w\left\{x \in \mathbb{R}^{n}:|T f(x)|>\lambda\right\} \leq c\|w\|_{A_{1}} \int_{\mathbb{R}^{n}}|f| w d x .
$$


We recall that $w$ is an $A_{1}$ weight if there is a finite constant $c$ such that $M w \leq c w$ a.e., and where $\|w\|_{A_{1}}$ denotes the smallest of these $c$. In a recent paper [11] we proved the following related result:

Theorem 1.3. Let $\varphi(t)=t\left(1+\log ^{+} t\right)\left(1+\log ^{+} \log ^{+} t\right)$. There is a constant $c=c(n, T)$ such that for any $A_{1}$ weight $w$ and for all $f \in$ $L_{w}^{1}\left(\mathbb{R}^{n}\right)$

$$
\sup _{\lambda>0} \lambda w\left\{x \in \mathbb{R}^{n}:|T f(x)|>\lambda\right\} \leq c \varphi\left(\|w\|_{A_{1}}\right) \int_{\mathbb{R}^{n}}|f| w d x .
$$

Analogously, we do not know whether a weak variant of (1.3) is true:

$$
\sup _{\lambda>0} \lambda w\left\{x \in \mathbb{R}^{n}: \frac{|T f(x)|}{w}>\lambda\right\} \leq c\|w\|_{A_{1}} \int_{\mathbb{R}^{n}}|f| d x .
$$

Theorems 1.1 and 1.2 show that in "dual" direction we have a worst result in terms of $M^{k}$. Therefore, it is natural to expect that the bound for the left-hand side of (1.4) in terms of $\|w\|_{A_{1}}$ must be at least not better than the one in Theorem 1.3. However, we prove the following surprising result.

Theorem 1.4. There is a constant $c=c(n, T)$ such that for any $A_{1}$ weight $w$ and for all $f \in L^{1}\left(\mathbb{R}^{n}\right)$,

$\sup _{\lambda>0} \lambda w\left\{x \in \mathbb{R}^{n}: \frac{|T f(x)|}{w}>\lambda\right\} \leq c\|w\|_{A_{1}}\left(1+\log ^{+}\|w\|_{A_{1}}\right) \int_{\mathbb{R}^{n}}|f| d x$.

The paper is organized as follows. Section 2 contains some preliminary information about maximal operators and singular integrals. Proofs of Theorems 1.2 and 1.4 are contained in Sections 3. Several concluding remarks are given in Section 4.

\section{Preliminaries}

2.1. Maximal Operator. Given a locally integrable function $f$ on $\mathbb{R}^{n}$, the Hardy-Littlewood maximal operator $M$ is defined by

$$
M f(x)=\sup _{Q \ni x} \frac{1}{|Q|} \int_{Q}|f(y)| d y,
$$

where the supremum is taken over all cubes $Q$ containing the point $x$.

It is well-known (see, e.g., [10, p. 175]) that

$$
M^{k} f(x) \asymp \sup _{Q \ni x} \frac{1}{|Q|} \int_{Q}|f| \log \left(\frac{|f|}{|f|_{Q}}+e\right)^{k-1} d y,
$$

where $f_{Q}=\frac{1}{|Q|} \int_{Q} f$. From this, by Hölder's inequality we obtain

$$
M^{k} f \leq c(M f)^{1-\frac{1}{k}}\left(M^{k+1} f\right)^{\frac{1}{k}} .
$$


In particular, for $k=2$ we have the following estimate

$$
\left(\frac{M f}{M^{3} f}\right)^{\frac{1}{2}} \leq c \frac{M f}{M^{2} f} .
$$

We say that a weight $w$ satisfies the $A_{p}$ condition if there exists a constant $c>0$ such that for any cube $Q$,

$$
\left(\int_{Q} w\right)\left(\int_{Q} w^{-1 /(p-1)}\right)^{p-1} \leq c|Q|^{p} .
$$

The smallest possible $c$ here is denoted by $\|w\|_{A_{p}}$. Set $A_{\infty}=\cup_{p \geq 1} A_{p}$. We recall that Muckenhoupt's theorem [12] says that the maximal operator $M$ is bounded on $L_{w}^{p}, 1<p<\infty$, if and only if $w \in A_{p}$.

We mention several well-known facts about $A_{p}$ weights. First, it follows from definitions and from Hölder's inequality that if $w_{1}$ and $w_{2}$ are $A_{1}$ weights, then $w_{1} w_{2}^{1-p} \in A_{p}$, and

$$
\left\|w_{1} w_{2}^{1-p}\right\|_{A_{p}} \leq\left\|w_{1}\right\|_{A_{1}}\left\|w_{2}\right\|_{A_{1}}^{p-1} .
$$

Next, if $0<\alpha<1$, then $(M f)^{\alpha} \in A_{1}$ (see [4]), and

$$
\left\|(M f)^{\alpha}\right\|_{A_{1}} \leq c_{n, \alpha} .
$$

Let $M_{w}^{c}$ be the weighted centered maximal operator defined by

$$
M_{w}^{c} f(x)=\sup _{Q \ni x} \frac{1}{w(Q)} \int_{Q}|f(y)| w(y) d y,
$$

where the supremum is taken over all cubes $Q$ centered at $x$. By the Besicovitch covering theorem,

$$
w\left\{x \in \mathbb{R}^{n}: M_{w}^{c} f(x)>\lambda\right\} \leq \frac{c_{n}}{\lambda}\|f\|_{L_{w}^{1}} .
$$

It is easy to see that for any $x \in \mathbb{R}^{n}$ one has

$$
M f(x) \leq c_{n} M_{w}^{c}(f / w)(x) M w(x) .
$$

This along with (2.5) implies the following.

Proposition 2.1. For any weight $w$ and for any $f \in L^{1}\left(\mathbb{R}^{n}\right)$,

$$
\left\|\frac{M f}{M w}\right\|_{L_{w}^{1, \infty}} \leq c\|f\|_{L^{1}}
$$


2.2. Calderón-Zygmund operators. Let $K(x, y)$ be a locally integrable function defined off the diagonal $x=y$ in $\mathbb{R}^{n} \times \mathbb{R}^{n}$, which satisfies the size estimate

$$
|K(x, y)| \leq \frac{c}{|x-y|^{n}}
$$

and, for some $\varepsilon>0$, the regularity condition

$$
|K(x, y)-K(z, y)|+|K(y, x)-K(y, z)| \leq c \frac{|x-z|^{\varepsilon}}{|x-y|^{n+\varepsilon}},
$$

whenever $2|x-z|<|x-y|$.

A linear operator $T: C_{0}^{\infty}\left(\mathbb{R}^{n}\right) \rightarrow L_{\text {loc }}^{1}\left(\mathbb{R}^{n}\right)$ is a Calderón-Zygmund operator if it extends to a bounded operator on $L^{2}\left(\mathbb{R}^{n}\right)$, and there is a kernel $K$ satisfying (2.6) and (2.7) such that

$$
T f(x)=\int_{\mathbb{R}^{n}} K(x, y) f(y) d y
$$

for any $f \in C_{0}^{\infty}\left(\mathbb{R}^{n}\right)$ and $x \notin \operatorname{supp}(f)$.

We shall need the following estimate due to $\mathrm{R}$. Coifman $[2,3]$ : for any $0<p<\infty$ and for $w \in A_{\infty}$,

$$
\int_{\mathbb{R}^{n}}|T f|^{p} w d x \leq c \int_{\mathbb{R}^{n}}(M f)^{p} w d x .
$$

The following theorem has been recently proved in [11].

Theorem 2.2. Let $1<p<\infty$ and let $\nu_{p}=\frac{p^{2}}{p-1} \log \left(e+\frac{1}{p-1}\right)$. There is a constant $c=c(n, T)$ such that for any $A_{1}$ weight $w$,

$$
\|T\|_{L^{p}(w)} \leq c \nu_{p}\|w\|_{A_{1}} .
$$

Let $T^{*}$ be the adjoint operator of $T$. Then $T^{*}$ is also a CalderónZygmund operator. Applying (2.9) to $T^{*}$ instead of $T$ and using duality we have that $(2.9)$ is equivalent to

$$
\|T\|_{L^{p^{\prime}\left(w^{1-p^{\prime}}\right)}} \leq c \nu_{p}\|w\|_{A_{1}}
$$

where, as usual, $1 / p+1 / p^{\prime}=1$.

\section{Proofs of MAIN Results}

Proof of Theorem 1.2. We start with some ideas used in [5]. Fix $p>1$. Let

$$
S f=\frac{M\left(f(M w)^{1-1 / 2 p}\right)}{(M w)^{1-1 / 2 p}} .
$$

Note that by (2.4) along with (2.3), a weight $(M w)^{1-r\left(1-\frac{1}{2 p}\right)}$ belongs to $A_{r}$ with corresponding constants independent of $w$. Hence, by the 
Muckenhoupt theorem [12], $S$ is bounded on $L_{M w}^{r}$ for any $r>1$. Therefore, by the Marcinkiewicz interpolation theorem [1, p. 225], $S$ is bounded on $L_{M w}^{p^{\prime}, 1}$.

We now apply the Rubio de Francia algorithm (see [9]) to define the operator $\mathcal{R}$ by

$$
\mathcal{R} h(x)=\sum_{j=0}^{\infty} \frac{S^{j} h(x)}{(2 K)^{j}}
$$

where $K$ is the "norm" of $S$ on $L_{M w}^{p^{\prime}, 1}$. It is easy to see that

(a) $h(x) \leq \mathcal{R} h(x)$

(b) $\|\mathcal{R} h\|_{L^{p^{\prime}, 1}(M w)} \leq 2\|h\|_{L^{p^{\prime, 1}(M w)}} ;$

(c) $S(\mathcal{R} h)(x) \leq 2 K \mathcal{R} h(x)$.

It follows from the last property that $(\mathcal{R} h)(M w)^{1-1 / 2 p} \in A_{1}$ with the $A_{1}$ constant bounded by $2 K$. Using this fact and since $\frac{M w}{M^{3} w} \leq 1$, we conclude by (2.3) that

$$
\frac{(\mathcal{R} h) M w}{\left(M^{3} w\right)^{\frac{1}{p}}} \leq \frac{(\mathcal{R} h)(M w)^{1-\frac{1}{2 p}}}{\left(M^{3} w\right)^{\frac{1}{2 p}}} \in A_{2}
$$

with the $A_{2}$ constant depending only on $p$ and $n$.

Observe now that for any $p>1$ we have

$$
\left\|\frac{|T f|}{M^{3} w}\right\|_{L_{w}^{1, \infty}} \leq\left\|\frac{|T f|}{M^{3} w}\right\|_{L_{M w}^{1, \infty}}=\left\|\left(\frac{|T f|}{M^{3} w}\right)^{1 / p}\right\|_{L_{M w}^{p, \infty}}^{p} .
$$

Next, by duality,

$$
\left\|\left(\frac{|T f|}{M^{3} w}\right)^{1 / p}\right\|_{L_{M w}^{p, \infty}}=\sup \int_{\mathbb{R}^{n}}|T f|^{\frac{1}{p}} h \frac{M w}{\left(M^{3} w\right)^{\frac{1}{p}}} d x,
$$

where the supremum is taken over all non-negative $h \in L_{M w}^{p^{\prime}, 1}$ with $\|h\|_{L_{M w}^{p^{\prime}, 1}}=1$. Applying (3.1) and (2.8), we obtain

$$
\begin{aligned}
\int_{\mathbb{R}^{n}}|T f|^{\frac{1}{p}} \frac{h M w}{\left(M^{3} w\right)^{\frac{1}{p}}} d x & \leq \int_{\mathbb{R}^{n}}|T f|^{\frac{1}{p}} \frac{(\mathcal{R} h) M w}{\left(M^{3} w\right)^{\frac{1}{p}}} d x \\
& \leq c \int_{\mathbb{R}^{n}} M f(x)^{\frac{1}{p}} \frac{(\mathcal{R} h)(M w)^{1-\frac{1}{2 p}}}{\left(M^{3} w\right)^{\frac{1}{2 p}}} d x .
\end{aligned}
$$


From the last estimate and from (2.2) we get

$$
\begin{aligned}
\int_{\mathbb{R}^{n}}|T f|^{\frac{1}{p}} h \frac{M w}{\left(M^{3} w\right)^{\frac{1}{p}}} d x & \leq c\left\|\left(\frac{M f}{M^{2} w}\right)^{1 / p}\right\|_{L^{p, \infty}(M w)}\|\mathcal{R} h\|_{L^{p^{\prime}, 1}(M w)} \\
& \leq 2 c\left\|\left(\frac{M f}{M^{2} w}\right)^{1 / p}\right\| \|_{L^{p, \infty}(M w)} \\
& =2 c\left\|\frac{M f}{M^{2} w}\right\|_{L^{1, \infty}(M w)}^{1 / p}
\end{aligned}
$$

(we have used also property (b) of $\mathcal{R} h$ and that $\|h\|_{L_{M w}^{p^{\prime}, 1}}=1$ ).

Applying Proposition 2.1 to the last inequality completes the proof.

Proof of Theorem 1.4. We follow here the classical method of CalderónZygmund although with some modifications. Fix $\lambda>0$, and set

$$
\Omega_{\lambda}=\left\{x \in \mathbb{R}^{n}: M_{w}^{c}(f / w)(x)>\lambda\right\} .
$$

Let $\cup_{j} Q_{j}$ be the Whitney covering of $\Omega_{\lambda}$. Set $b(x)=\sup _{j}\left(f-f_{Q_{j}}\right) \chi_{Q_{j}}(x)$ and $g(x)=f(x)-b(x)$.

By (2.5),

$$
w\left(\Omega_{\lambda}\right) \leq \frac{c_{n}}{\lambda}\|f\|_{L^{1}}
$$

Hence, we have to estimate

$$
\begin{aligned}
w\left\{x \notin \Omega_{\lambda}: \frac{|T f|}{w}>\lambda\right\} & \leq w\left\{x \notin \Omega_{\lambda}: \frac{|T b|}{w}>\lambda / 2\right\} \\
& +w\left\{x \notin \Omega_{\lambda}: \frac{|T g|}{w}>\lambda / 2\right\} \equiv I_{1}+I_{2} .
\end{aligned}
$$

By [7, Ineq. (5.4), p. 92] or [9, Lemma 5, p. 413], we get

$$
I_{1} \leq \frac{2}{\lambda} \int_{\mathbb{R}^{n} \backslash \Omega_{\lambda}}|T b(x)| d x \leq \frac{c}{\lambda} \sum_{j} \int_{Q_{j}}\left|f-f_{Q_{j}}\right| d x \leq \frac{c}{\lambda}\|f\|_{L^{1}},
$$

where $c=c(T, n)$.

Applying (2.10) for $1<p^{\prime}<2$ yields

$$
\begin{aligned}
I_{2} & \leq \frac{c p^{p^{\prime}}\|w\|_{A_{1}}^{p^{\prime}}}{\lambda^{p^{\prime}}} \int_{\mathbb{R}^{n}}|g|^{p^{\prime}} w^{1-p^{\prime}} d x \\
& \leq \frac{c p^{p^{\prime}}\|w\|_{A_{1}}^{p^{\prime}}}{\lambda^{p^{\prime}}}\left(\int_{\mathbb{R}^{n} \backslash \Omega_{\lambda}}|f|^{p^{\prime}} w^{1-p^{\prime}} d x+\sum_{j}\left(|f|_{Q_{j}}\right)^{p^{\prime}} \int_{Q_{j}} w^{1-p^{\prime}} d x\right) .
\end{aligned}
$$


We have that $|f| \leq c w$ a.e. on $\mathbb{R}^{n} \backslash \Omega_{\lambda}$, and hence

$$
\int_{\mathbb{R}^{n} \backslash \Omega_{\lambda}}|f|^{p^{\prime}} w^{1-p^{\prime}} d x \leq \lambda^{p^{\prime}-1}\|f\|_{L^{1}}
$$

Next, by properties of the Whitney covering, it is easy to see that for any cube $Q_{j}$ there exists a cube $Q_{j}^{*}$ such that $Q_{j} \subset Q_{j}^{*},\left|Q_{j}^{*}\right| \leq c_{n}\left|Q_{j}\right|$, and the center of $Q_{j}^{*}$ lies outside of $\Omega_{\lambda}$. Therefore,

$$
\begin{aligned}
& \left(|f|_{Q_{j}}\right)^{p^{\prime}-1} \int_{Q_{j}} w^{1-p^{\prime}} d x \leq\|w\|_{A_{1}}^{p^{\prime}-1}\left(|f|_{Q_{j}}\right)^{p^{\prime}-1} \int_{Q_{j}}(M w)^{1-p^{\prime}} d x \\
& \leq c\|w\|_{A_{1}}^{p^{\prime}-1}\left|Q_{j}\right|\left(\frac{|f|_{Q_{j}^{*}}}{w_{Q_{j}^{*}}}\right)^{p^{\prime}-1} \leq c\left(\lambda\|w\|_{A_{1}}\right)^{p^{\prime}-1}\left|Q_{j}\right|,
\end{aligned}
$$

which gives

$$
\begin{aligned}
\sum_{j}\left(|f|_{Q_{j}}\right)^{p^{\prime}} \int_{Q_{j}} w^{1-p^{\prime}} d x & \leq c\left(\lambda\|w\|_{A_{1}}\right)^{p^{\prime}-1} \sum_{j}|f|_{Q_{j}}\left|Q_{j}\right| \\
& \leq c\left(\lambda\|w\|_{A_{1}}\right)^{p^{\prime}-1}\|f\|_{L^{1}} .
\end{aligned}
$$

Combining the previous estimates, we obtain

$$
I_{2} \leq \frac{c p^{p^{\prime}}\|w\|_{A_{1}}^{2 p^{\prime}-1}}{\lambda}\|f\|_{L^{1}}
$$

Choose now $p^{\prime}=1+\frac{1}{\log \left(e+\|w\|_{A_{1}}\right)}$. Then we get

$$
I_{2} \leq \frac{c\|w\|_{A_{1}} \log \left(e+\|w\|_{A_{1}}\right)}{\lambda}\|f\|_{L^{1}} .
$$

This, along with estimates for $I_{1}$ and for $w\left(\Omega_{\lambda}\right)$, completes the proof.

\section{Concluding Remarks}

Remark 4.1. Following (2.1), we extend the definition of $M^{k}$ to fractional order, setting for any $\alpha \geq 1$,

$$
M^{\alpha} f(x)=\sup _{Q \ni x} \frac{1}{|Q|} \int_{Q}|f| \log \left(\frac{|f|}{|f|_{Q}}+e\right)^{\alpha-1} d y .
$$

It was shown in [16] that $M^{2} w$ in Theorem 1.1 can be replaced by $M^{1+\varepsilon} w$ for any $\varepsilon>0$ (with corresponding constant depending on $\varepsilon$ ).

Similarly, one can show that $M^{3} w$ in Theorem 1.2 can be replaced by $M^{2+\varepsilon} w$ for any $\varepsilon>0$. 
Remark 4.2. It is easy to see that actually in Theorem 1.2 we proved a stronger inequality, namely,

$$
\sup _{\lambda>0} \lambda M w\left\{x \in \mathbb{R}^{n}: \frac{|T f(x)|}{M^{3} w}>\lambda\right\} \leq c \int_{\mathbb{R}^{n}}|f| d x .
$$

This yields an additional indication that Theorem 1.2 should be true with $M^{2} w$ instead of $M^{3} w$.

Remark 4.3. Combining ideas used in the proving Theorem 1.4 with some estimates obtained in [16], one can show that Theorem 1.2 can be improved by replacing $M^{3} w$ by a smaller weight $\left(M^{3} w\right)^{1-\varepsilon} w^{\varepsilon}$ for any $0<\varepsilon<1 / 2$. We emphasize, however, that a more principal question of interest if $M^{3} w$ can be replaced by $M^{2} w$ or simply by $M w$.

\section{REFERENCES}

[1] C. Bennett and R. Sharpley, Interpolation of operators, Academic Press, New York, 1988.

[2] R.R. Coifman, Distribution function inequalities for singular integrals, Proc. Nat. Acad. Sci. USA, 69 (1972), 2838-2839.

[3] R.R. Coifman and C. Fefferman, Weighted norm inequalities for maximal functions and singular integrals, Studia Math. 51 (1974), 241-250.

[4] R.R. Coifman and R. Rochberg, Another characterization of BMO, Proc. Amer. Math. Soc., 79 (1980), 249-254.

[5] D. Cruz-Uribe, J.M. Martell and C. Pérez, Weighted weak-type inequalities and a conjecture of Sawyer, Int. Math. Res. Not., 30 (2005), 1849-1871.

[6] D. Cruz-Uribe and C. Pérez, Two-weight extrapolation via the maximal operator, J. Funct. Anal., 174 (2000), no. 1, 1-17.

[7] J. Duoandikoetxea, Fourier Analysis, Grad. Studies Math. 29, Amer. Math. Soc., Providence, 2000.

[8] C. Fefferman and E.M. Stein, Some maximal inequalities, Amer. J. Math., 93 (1971), 107-115.

[9] J. García-Cuerva and J.L. Rubio de Francia, Weighted norm inequalities and related topics, North-Holland Math. Stud. 116, North-Holland, Amsterdam, 1985.

[10] T. Iwaniec and G. Martin, Geometric function theory and non-linear analysis, Oxford University Press, 2001.

[11] A.K. Lerner, S. Ombrosi and C. Pérez, Sharp $A_{1}$ bounds for Calderón-Zygmund operators and the relationship with a problem of Muckenhoupt-Wheeden, preprint. Available at http://www.math.biu.ac.il/ lernera/Publications.html

[12] B. Muckenhoupt, Weighted norm inequalities for the Hardy maximal function, Trans. Amer. Math. Soc. 165 (1972), 207-226.

[13] B. Muckenhoupt and R. Wheeden, personal communication.

[14] B. Muckenhoupt and R. Wheeden, Some weighted weak-type inequalities for the Hardy-Littlewood maximal function and the Hilbert transform, Indiana Univ. Math. J. 26 (1977), 801-816. 
[15] S. Ombrosi y C. Pérez, Mixed weak type estimates: Examples and counterexamples related to a problem of E. Sawyer, preprint.

[16] C. Pérez, Weighted norm inequalities for singular integral operators, J. London Math. Soc., 49 (1994), 296-308.

[17] E.T. Sawyer, A weighted weak type inequality for the maximal function, Proc. Amer. Math. Soc. 93 (1985), 610-614.

Departamento de Análisis Matemático, Facultad de Matemáticas, Universidad De SeVIlla, 41080 Sevilla, Spain

E-mail address: aklerner@netvision.net.il

Departamento de Matemática, Universidad Nacional del Sur, Bahía Blanca, 8000, Argentina

Current address: Departamento de Análisis Matemático, Facultad de Matemáticas, Universidad de Sevilla, 41080 Sevilla, Spain

E-mail address: sombrosi@uns.edu.ar

Departamento de Análisis Matemático, Facultad de Matemáticas, Universidad De Sevilla, 41080 Sevilla, Spain

E-mail address: carlosperez@us.es 